\title{
Predicting hydrogen isotope inventory in plasma-facing components during normal and abnormal operations in fusion devices
}

\author{
Alice $\mathrm{Hu}^{*}$ and Ahmed Hassanein \\ Center for Materials under Extreme Environment, School of Nuclear Engineering \\ Purdue University, West Lafayette, IN 47906, USA
}

\begin{abstract}
Hydrogen isotope behavior and inventory in plasma-facing components (PFCs) of fusion devices are key concerns for safe, reliable, and economical operation. To accurately estimate hydrogen isotope retention and recovery in tungsten (the current leading candidate as a PFC), we have developed a model that was recently benchmarked against isotope depth profile and retention level in a tungsten target under various conditions and compared with both experimental data and simulation results. In this research, we have extended the model to include details of transient events. Therefore, one can use this model to estimate hydrogen isotope retention behavior in tungsten and potential other PFC candidates during normal operational pulse, effects of edge-localized modes (ELMs), and a possible cleaning processes scenario.
\end{abstract}

Keywords: fusion devices, plasma-facing components, ITER, tritium retention, ELMs

Email address: alicehu@ cityu.edu.hk

*Current Address: City University of Hong Kong, Hong Kong

1. Introduction

In future thermonuclear fusion experiments, such as those in the International Thermonuclear Experimental Reactor (ITER), removal of hydrogen isotopes from plasma-facing components (PFCs) is a key aspect of reliable operation. Fuel loss, safety, and wall recovery issues are key engineering challenges in fusion reactor design because tritium is expensive, in short supply, radioactive, and has safety issues. Tungsten has become the leading candidate material in fusion reactor PFCs due to its low sputtering yield and high melting point. However, the quantitative prediction of tritium retention in tungsten under ITER conditions remains difficult [1].

Because hydrogen isotope retention and migration in tungsten has become a crucial problem, the transport and trapping properties of hydrogen isotopes in tungsten are critical and require further understanding. Figure 1 in Refs. $[2,3]$ shows a common hydrogen isotope spatial profile in metal. Spatial distribution of hydrogen isotopes in metal can be generally divided into three regions: near surface (I), subsurface (II), and bulk (III). Region I has the highest concentration because incoming hydrogen is implanted and trapped in a layer only a few nanometers thick. Region II shows the signature peak distribution of atomic diffusion in a metal lattice. In Region III, the concentration extends and diffuses gradually into the bulk and becomes less intense. In order to explain such a spatial profile and the "uphill diffusion" in Region II, the classical Gorsky effect, which in sample material contains a stress field induced by external bending, has been modified to contain a stress field induced by internal lattice distortion and was included in our previous work [3]. Worth to mention that there is no tensile strain in this deuterium irradiation on tungsten case compared to classical Gorsky effect. The bending scenario of Gorsky effect can result in one side of compressive strain and the other side of tensile strain. However in this work, we proposed that the massive amount of deuterium implantation on the surface will pressurize, distort neighbor lattices, and produce a high compressive stress area beneath the oversaturated implantation peak. The compressive stress zone will gradually decrease and relax, as extending into the bulk leaving only compressive strain area.

(C) 2015. This manuscript version is made available under the Elsevier user license http://www.elsevier.com/open-access/userlicense/1.0/ 


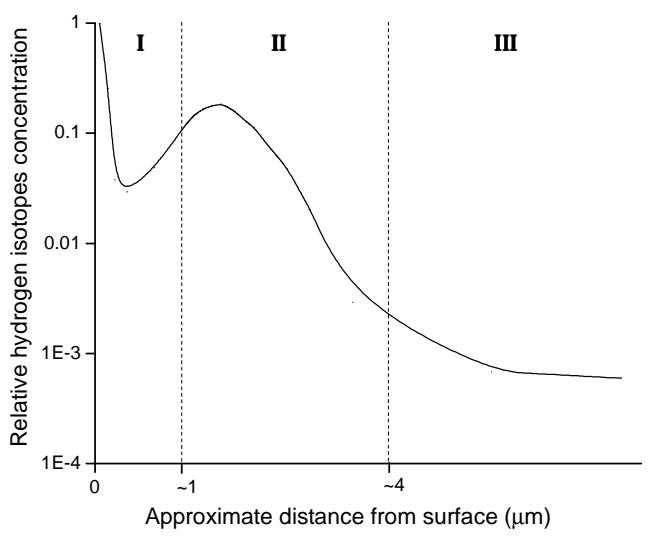

Figure 1. Schematic diagram of hydrogen isotope depth profile in metals $[2,3]$

Such high irradiation process is extreme since normally hydrogen isotopes will diffuse into metal and will not accumulate on metal surface. The deuterium concentration in the implantation zone, under this low energy high flux/fluence irradiation condition, greatly exceeds the solubility limit and stresses the matrix lattice until plastic deformation occurs. Results from previous research [3] allow us to benchmark our model with other experimental and simulation results and establish agreement with other studies. In this paper, we will demonstrate possible further applications to our model. Figure 2 schematically illustrates the accumulated hydrogen isotope wall inventory during operation, including the effect of edge-localized modes (ELMs) with certain frequencies and long ITER pulse operation.

The retained fraction during the pulsed operation can be given by

$R_{\text {pulse }}=\frac{N_{\text {wall }}}{N_{\text {inj }}}$

where $N_{\text {wall }}$ is the wall inventory accumulated during the pulse and $N_{i n j}$ is the integrated particle injected in the wall. The retained fraction after the ELMs can be represented by

$R_{E L M s}=\frac{N_{w a l l}-N_{E L M S}}{N_{i n j}}$

where $N_{E L M S}$ is the hydrogen-isotope particle released after ELMs. The retained fraction can then be given by

$R_{\text {short }}=\frac{N_{\text {wall }}-N_{E L M S}-N_{\text {rec }}}{N_{\text {inj }}}$

where $N_{\text {rec }}$ is particle recovery/release after the end of the shot. Finally, the long-term retention, including wall inventory accumulated during the pulse $N_{\text {wall }}$, integrated particle injected in wall $N_{\text {inj }}$, hydrogen-isotope particle released after ELMs $N_{E L M S}$, particle recovery/release after the end of the shot $N_{\text {rec }}$, recovery overnight $N_{\text {night }}$, recovery during cleaning (isotope removal) procedures $N_{\text {clean }} \ldots$... etc., can be expressed as

$R_{\text {long }}=\frac{\sum\left(N_{\text {wall }}-N_{E L M S}-N_{\text {rec }}-N_{\text {night }}-N_{\text {clean } \cdots)}\right)}{N_{\text {inj }}}$

The goal of this work is quantitative determination of the retained amount from a regular pulse during operation, as well as how the ELM and cleaning (removal) process will affect the hydrogen isotope retention level. 


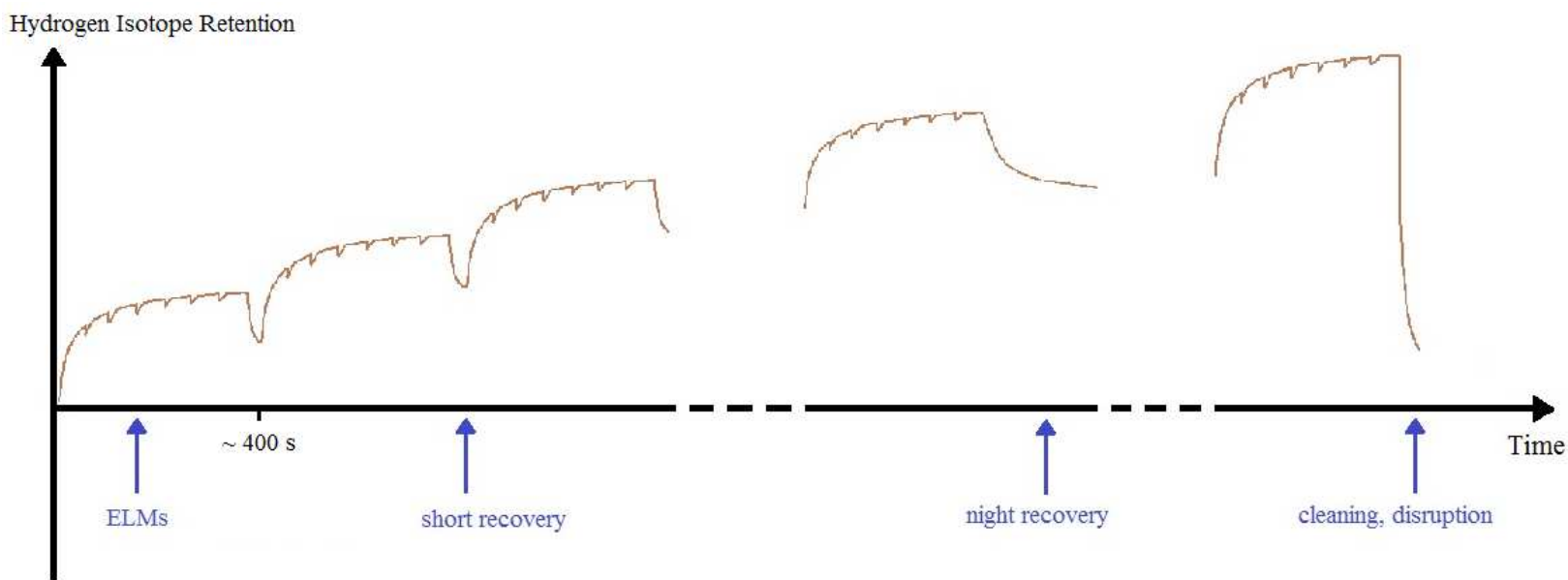

Figure 2. Schematic view of particle balance, showing wall inventory buildup during a shot and recovery after the shot. Recovery after cleaning procedures and/or a disruption is also illustrated

A critical issue for ITER is minimizing fuel inventory and safely removing hydrogen isotopes from PFCs without shutting down the reactor, venting the vacuum vessel, deconditioning the plasma-facing surfaces, and causing excessive exposure to radioactive materials. Two main methods are now available for overnight cleaning and removal of hydrogen isotope from PFCs. The first is in-situ detritiation by oxygen treatment to produce $\mathrm{T}_{2} \mathrm{O} / \mathrm{DTO}$ [4]. The second method uses continuous-wave Nd laser beam scanning at high speed to heat the walls to higher temperatures (e.g., $2300 \mathrm{~K}$ ) for short durations (e.g., $10 \mathrm{~ms}$ ). Skinner et al. [5] reported that the laser cleaning method can remove up to $87 \%$ of the tritium from JET (Joint European Torus) PFCs, which are composed of carbon and beryllium. Although the laser detritiation method is not part of the current ITER plan and we consider tungsten instead of carbon and beryllium in our research, we will use intense laser heating, i.e., the $2300 \mathrm{~K}$ cleaning for $10 \mathrm{~ms}$, in our simulations to evaluate the effectiveness of the method in hydrogen isotope removal.

2. Model and Methods

2.1 Governing equations

We have developed an integrated model to describe hydrogen isotope behavior in tungsten based on the Gorsky effect and a general chemical potential as described in Ref. 3. The conditions treated here are extreme and justifies the use of this effect. The diffusion processes are not only related to concentration but can also be significantly affected by stress and temperature distribution. Because the experimental coefficient values for tritium are not yet available, we use deuterium for simulating hydrogen isotope diffusion in tungsten. The bombardment and the depth profiles of $200 \mathrm{eV}$ deuterium in single-crystal tungsten have been modeled and compared with recent work. Total deuterium retention at various temperatures and fluences are also simulated and compared with available data. The results are in reasonable agreement with data [6], thus allowing use of this model to estimate deuterium/tritium inventory and recovery in PFCs.

The major equations used in our model are [3]:

$$
\begin{aligned}
& \frac{d C}{d t}=G+D \frac{\partial^{2} C}{\partial x^{2}}+\frac{D C \bar{V}_{H}}{R T} \frac{\partial^{2} p}{\partial x^{2}}-\sum_{i} \frac{d C_{T i}}{d t} \\
& \frac{d C_{T i}}{d t}=\frac{D}{\lambda^{2}} C \frac{C_{T r a p i}^{0}-C_{T i}}{W}-C_{T i} \frac{D_{0}}{\lambda^{2}} \exp \left(\frac{-\mathrm{E}_{\mathrm{Ti}}}{\mathrm{kT}}\right) \\
& \frac{\partial T}{\partial t}=\alpha \frac{\partial^{2} T}{\partial x^{2}}
\end{aligned}
$$

where $\mathrm{C}$ is mobile deuterium concentration; $\mathrm{C}_{\mathrm{T}}$ is total deuterium concentration; $\mathrm{C}_{\mathrm{Ti}}$ is the i-th trapped deuterium atomic concentration; $\mathrm{T}$ is temperature; $\mathrm{G}$ is the implanted deuterium flux in normal distribution form; and $\mathrm{D}$ is the 
diffusion coefficient. $\mathrm{D}_{0}$ is the diffusion pre-exponential factor. Diffusion rate is faster in the near-surface region due to enhancement from radiation-induced effects [7]; $\bar{V}_{H}$ is the partial molar volume (the change in volume per mole of hydrogen added to the solid solution); $\mathrm{R}$ is the universal gas constant; $\mathrm{p}$ is the equivalent pressure stress; $\lambda$ is the jump distance; $C_{\text {Trapi }}^{0}$ is the maximum trapping density, which are given in Reference [7]; $\mathrm{W}$ is tungsten atomic density; $\mathrm{E}_{\mathrm{Ti}}$ is the $\mathrm{i}$-th detrapping energy, corresponding to $0.85 \mathrm{eV}$ (dislocation sites and grain boundaries) and 1.45 $\mathrm{eV}$ (deuterium cluster and vacancy trapping); $\mathrm{k}$ is the Boltzmann constant; and $\alpha$ is thermal diffusivity. This system of partial differential equations is reduced to ordinary differential equations by the finite-difference method and solved using SUNDIALS [8], where Eq. (5) stands for the change in concentration for mobile deuterium, including implantation flux G, diffusion by concentration gradient $D \frac{\partial^{2} C}{\partial x^{2}}$, diffusion by stress gradient $\frac{D C \bar{V}_{H}}{R T} \frac{\partial^{2} p}{\partial x^{2}}$ and deuterium amount $\sum_{i} \frac{d C_{T i}}{d t}$ that become detrapped; Eq. (6) represents the change in trapped deuterium concentration, including amount of mobile deuterium that is trapped $\frac{D}{\lambda^{2}} C \frac{C_{T r a p i}^{0}-C_{T i}}{W}$ and the amount of trapped deuterium $C_{T i} \frac{D_{0}}{\lambda^{2}} \exp \left(\frac{-\mathrm{E}_{\mathrm{Ti}}}{\mathrm{kT}}\right)$ become mobile; and Eq. (7) describes the transient temperature distribution. More detailed numerical values of each coefficient are listed in Ref. 3.

From the Maxwell-Boltzmann distribution, when there are $\mathrm{N}$ atoms in matrix, the number of atoms having a Gibbs free energy $\mathrm{G}$ greater than $\mathrm{G}_{2}$ is $n\left(G>G_{2}\right)=N \exp \left(-G_{1} / k T\right)$. If we assume the energy $\mathrm{G}_{1}$ as a minimum energy datum, such that $n\left(G>G_{1}\right)=N$, then

$\frac{n\left(G>G_{2}\right)}{N}=\exp \left(-\frac{G_{2}-G_{1}}{k T}\right)=\exp \left(\frac{-\Delta G}{k T}\right)$

that is, the ratio of atoms with energy $\mathrm{G}_{2}$ at temperature $\mathrm{T}$.

There are several mechanisms for diffusion. For deuterium diffusion in tungsten, because deuterium is much smaller than tungsten, interstitial diffusion dominates. In the following, we used the interstitial mechanism to derive the diffusion coefficient $\mathrm{D}$. Assume $v$ is atom thermal vibration frequency, $\mathrm{z}$ is the interstitial coordinate number, $\lambda$ is lattice distance, and the physical meaning of jump frequency $\Gamma$ can be stated as $v \operatorname{zexp}\left(\frac{-\Delta G}{k T}\right)$. By definition, Gibbs free energy is $\Delta G=\Delta H-T \Delta S \approx \Delta U-T \Delta S$, where $\mathrm{H}$ is enthalpy, $\mathrm{T}$ is temperature, $\mathrm{S}$ is entropy, and $\mathrm{U}$ is internal energy. Thus,

$\Gamma=v \operatorname{zexp}\left(\frac{\Delta S}{k T}\right) \exp \left(\frac{-\Delta U}{k T}\right)$

and we obtain

$D=\lambda^{2} P \Gamma=\lambda^{2} P \nu \operatorname{zexp}\left(\frac{\Delta S}{k T}\right) \exp \left(\frac{-\Delta U}{k T}\right)=D_{0} \exp \left(\frac{-\Delta U}{k T}\right)$

where $D_{0}=\lambda^{2} P v \operatorname{zexp}\left(\frac{\Delta S}{k T}\right)$

Therefore, one can see that the physical meaning of $\frac{D}{\lambda^{2}}$ or $\frac{D_{0}}{\lambda^{2}}$ in Eq. (6) is in fact proportional to (a) the probability that deuterium would jump to the neighborhood spot and (b) the jump frequency.

The increase in trap density can be written as[7, 9](7, 9)(Ogorodnikova, Roth, \& Mayer, 2003; Ogorodnikova et al., 2008)(Ogorodnikova, Roth et al. 2003, Ogorodnikova, Roth et al. 2008) ${ }^{7,9}(7,9)^{7,9}(7,9)[7,9]^{7,97,9}$ (Ogorodnikova, Roth e Mayer, 2003; 2008):

$\frac{\mathrm{dC}_{\text {Trap }}}{\mathrm{dt}}=(1-\mathrm{r}) \cdot$ flux $\cdot \psi(x) \cdot \eta \cdot\left(1-\frac{\mathrm{C}_{\text {Trap }}}{\mathrm{C}_{\text {Trap }}^{0}}\right)$

where $\mathrm{C}_{\text {Trap }}$ is the trap density; $\mathrm{r}$ is the reflection coefficient; $\psi(x)$ is the depth distribution of ion-induced defects; $\eta$ is the defect creation rate, and $C_{\text {Trap }}^{0}$ is the maximum defect concentration. After integration with time t, trap density is obtained as: 
$C_{\text {Trap }}(x, t)=C_{\text {Trap }}^{0}\left(1-\exp \left(-(1-r) \cdot f l u x \cdot \psi(x) \cdot \eta \cdot \frac{t}{C_{\text {Trap }}^{0}}\right)\right)$

Four trap types are used; Following Ref. [7], two intrinsic traps (the dislocations have detrapping energy $0.85 \mathrm{eV}$ and the vacancies have detrapping energy $1.45 \mathrm{eV}$ ), and two ion-induced traps (the near-surface ion-induced trap has detrapping energy $1.45 \mathrm{eV}$ and the other ion-induced trap with wider range has detrapping energy $0.85 \mathrm{eV}$ ). The effect of annealing of the defects during the high temperatures is ignored during the short ELM events. Therefore, this trap profile evolution in respect to annealing needs to be further considered in future studies.

Regarding the input of Gorsky effect, from the research in hydrogen cracking in metals, the pressure value around the crack tip may range from hundreds to one thousand MPa [10,11]. Although the exact pressure induced by the stress field from Trapped-induced Gorsky effect (TGE) at the surface is unknown, we can safely assume that it is lower than the pressure around the crack tip. Thus we used a normal distribution with peak value of about $10 \mathrm{MPa}$ for simplification to evaluate the effect of pressure on the diffusion processes. The values of coefficients are listed in Table 1.

Table 1: Coefficient values

\begin{tabular}{|c|c|c|}
\hline & Meaning & Value \\
\hline $\mathrm{D}$ & diffusivity & $\mathrm{D}_{0} \times \exp \left(-\mathrm{E}_{\mathrm{d}} / \mathrm{kT}\right)$ \\
\hline $\mathrm{D}_{0}$ & Pre-exponential diffusion coefficient & $1.5 \times 10^{-10} \mathrm{~m}^{2} \mathrm{~s}^{-1}$ \\
\hline $\mathrm{E}_{\mathrm{d}}$ & migration energy & $0.39 \mathrm{eV}$ \\
\hline $\mathrm{K}_{\mathrm{r}}$ & Surface recombination coefficient & $\mathrm{K}_{\mathrm{r} 0} \times \exp \left(-\mathrm{E}_{\mathrm{r}} / \mathrm{kT}\right)$ \\
\hline $\mathrm{K}_{\mathrm{r} 0}$ & Pre-exponential recombination coefficient & $3.2 \times 10^{-15} \mathrm{~m}^{4} \mathrm{~s}^{-1}$ \\
\hline $\mathrm{Er}$ & Surface recombination energy & $1.16 \mathrm{eV}$ \\
\hline $\mathrm{W}$ & Tungsten atomic density & $6.3057 \times 10^{28} \mathrm{~m}^{-3}$ \\
\hline $\mathrm{D}_{\mathrm{m}}$ & Maximum diffusion coefficient & $10 \times \mathrm{D}$ \\
\hline$\lambda$ & jump distance & $3 \times 10^{-10} \mathrm{~m}$ \\
\hline$\overline{\mathrm{V}}_{\mathrm{H}}$ & Partial molar volume & $1.68 \times 10^{-6} \mathrm{~m}^{3} / \mathrm{mol}$ \\
\hline$\overline{\mathrm{E}_{\mathrm{T}}}$ & detrapping energy & $0.85,1.45 \mathrm{eV}$ \\
\hline \multirow[t]{4}{*}{$C_{\text {Trap }}^{0}$} & $0.85 \mathrm{eV}$ maximum intrinsic traps & $3 \times 10^{-5}$ at fr. \\
\hline & $1.45 \mathrm{eV}$ maximum intrinsic traps & $1 \times 10^{-5}$ at fr. \\
\hline & $0.85 \mathrm{eV}$ maximum ion-induced traps & 0.005 at fr. \\
\hline & $1.45 \mathrm{eV}$ maximum ion-induced traps & 0.01 at $\mathrm{fr}$ \\
\hline \multirow[t]{2}{*}{$\eta$} & $0.85 \mathrm{eV}$ ion-induced defect production rate & $2 \times 10^{-3}$ \\
\hline & $1.45 \mathrm{eV}$ ion-induced defect production rate & $1 \times 10^{-3}$ \\
\hline
\end{tabular}

\subsection{Simulation conditions}

Several different designs are available for the ITER inner surface tiles, including monoblock or flat tile design with carbon-fiber composites (CFC) or a tungsten surface. Current design for the divertor plate uses tungsten as the PFC on top of $\mathrm{CuCrZr}$ as the heat-sink material. The current first wall design is composed of beryllium material. 
However, in this analysis we consider and compare tungsten performance for both divertor and first wall material (this is an option for a future "all-tungsten" design machine. Because we focus on deuterium behavior in tungsten, the condition we model is the monoblock tungsten tile design (see Fig. 3).

To determine the scale and boundary condition for the simulation, we used Ref. $[12,13]$ to implement the initial and boundary conditions in our models. The initial conditions for deuterium concentration are zero everywhere before the initial pulse, and the starting temperature is distributed uniformly at $380 \mathrm{~K}$. Total length for simulating deuterium concentration is $200 \mu \mathrm{m}$ where the deuterium is not expected to penetrate or diffuse beyond that, while for temperature it is $15 \mathrm{~mm}$ where the coolant tubes reside beneath the structure. The boundary condition at the front surface for concentration equations is given by the hydrogen isotope current leaving the surface as:

$\mathrm{J}=\mathrm{K}_{\mathrm{r}} \mathrm{C}(0)^{2}$

We included D losses from the surface by taking molecular surface recombination coefficient into account, where $\mathrm{C}(0)$ is the hydrogen isotope concentration at the surface exposed to the plasma and $\mathrm{K}_{\mathrm{r}}$ is the surface recombination coefficient of atomic hydrogen isotope to molecules. The boundary conditions at the rear side is given by $\mathrm{C}=0$ or $\mathrm{J}$ $=0$. For the heat equation, the front-side temperature may vary according to edge-localized mode (ELM) intensity and duration of abnormal events, while the rear-side temperature is assumed constant at $380 \mathrm{~K}$, as determined from coolant conditions.
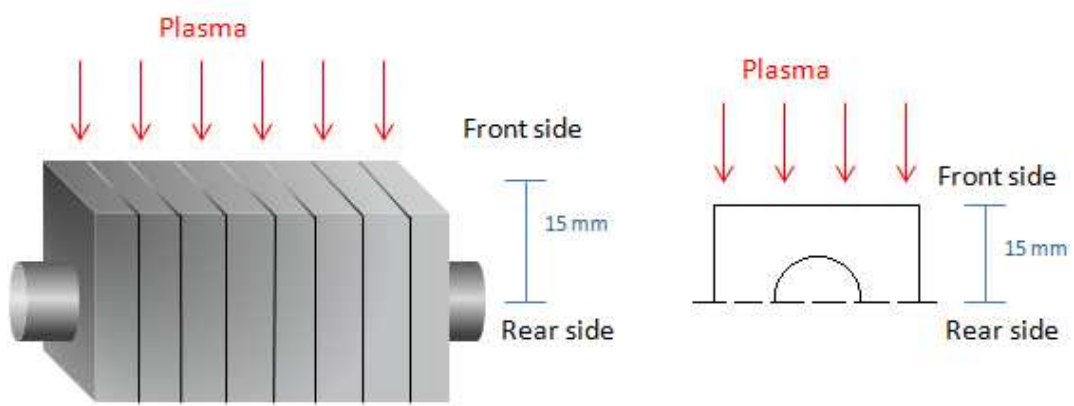

Figure 3. Typical ITER monoblock tungsten tile configuration and cross sectional view

The ELMs will release pulses of plasma particles to the first wall and divertor during reactor operation. Type I ELMs are considered in this research with frequencies 1 and $10 \mathrm{~Hz}$. These pulses will raise the temperatures of the PFC and wall and can affect hydrogen isotope retention. Deuterium flux to various PFCs is usually in the range of $10^{19}$ to $10^{21} \mathrm{~m}^{-2} \mathrm{~s}^{-1}$, with typical energy of about 100 to $300 \mathrm{eV}$ [14]. For prediction of hydrogen isotope retention in PFCs during these events, we assumed a flux value in our simulation of $10^{20} \mathrm{~m}^{-2} \mathrm{~s}^{-1}$ and a deuterium energy of $200 \mathrm{eV}$ for the first wall simulation [15], while the flux for the divertor is $10^{24} \mathrm{~m}^{-2} \mathrm{~s}^{-1}$ and deuterium energy is about $15 \mathrm{eV}$ [14]. Because this is a one-dimensional simulation, we consider only the temperature changes on the surface end, and for simplification, exclude the laser details such as wetted area, pulse shape, or energy. The deuterium depth profile was benchmarked against Alimov et al. [6] data, which after polishing the samples were annealed at $1573 \mathrm{~K}$ for 3.5 hours in a vacuum chamber with a background pressure of $\sim 2 * 10^{-4} \mathrm{~Pa}$ during the annealing process. The trap profile description and parameters were implemented from Ogorodnikova et al. [7] work. Their samples were annealed at $1573 \mathrm{~K}$ for 3 hours in vacuum with a background pressure of $2 * 10^{-4} \mathrm{~Pa}$ before implantation. Since both annealing conditions are similar, it is more straightforward to relate the experimental and simulation parameters together. The trap profiles will dynamically evolute during ion beam implantation, but assumed not to be influenced by annealing during our simulation of the short ELM transient events. As summarized in Table 2, temperature for the first-wall surface is assumed to be $500 \mathrm{~K}$, and divertor surface temperature is $1000 \mathrm{~K}$; also, ELM duration is $500 \mu$ s for each pulse and a single event is shown in Fig. 4; ELM temperature for the first wall is assumed to be $1500 \mathrm{~K}$ and for the divertor is $2000 \mathrm{~K}$, while that for the rear side is $380 \mathrm{~K}$. 
Table 2: Parameter values

\begin{tabular}{|l|l|l|}
\hline & First wall & Divertor \\
\hline Flux & $10^{20} \mathrm{~m}^{-2} \mathrm{~s}^{-1}$ & $10^{24} \mathrm{~m}^{-2} \mathrm{~s}^{-1}$ \\
\hline D energy & $200 \mathrm{eV}(8-300 \mathrm{eV})$ & $15 \mathrm{eV}$ \\
\hline Surface T & $500 \mathrm{~K}$ & $1000 \mathrm{~K}$ \\
\hline ELM pulse T & $1500 \mathrm{~K}$ & $2000 \mathrm{~K}$ \\
\hline
\end{tabular}

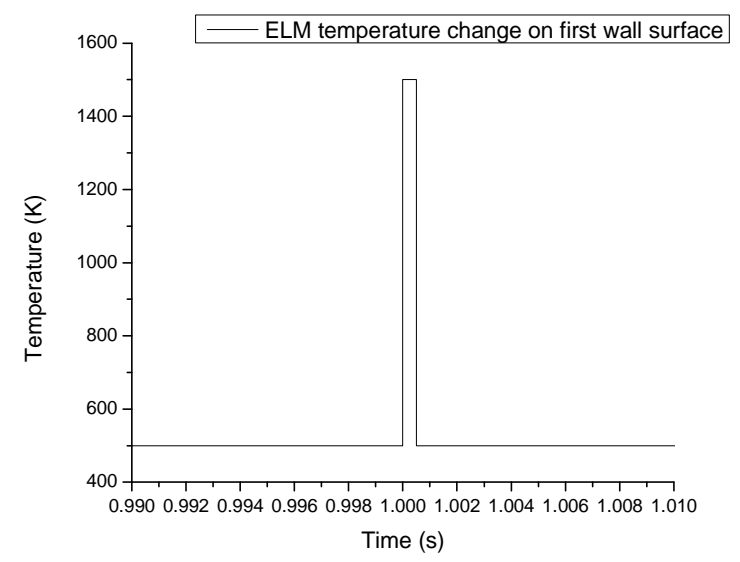

Figure 4. Example of the temperature rise during a single ELM event

\section{Results}

Modeling of detail hydrogen isotope retention during operation, which is illustrated in Fig. 1, is an important topic. In Fig. 5 we show retention level vs. time for three pulses each and $200 \mathrm{eV}$ deuterium with flux $1 \times$ $10^{20} \mathrm{~m}^{-2} \mathrm{~s}^{-1}$ at a $300 \mathrm{~K}$ tungsten target. The first pulse duration time is 0 to $400 \mathrm{~s}$, the second is 2000 to $2400 \mathrm{~s}$, and the third is 4000 to $4400 \mathrm{~s}$. As shown in Fig. 5, retention builds up during pulse duration and recovers when no flux is coming in. Also, retention increases with additional pulse times. There are no small ripples on each operational pulse (as shown in Fig. 2) because ELM is not considered here, but ripples will be shown in subsequent scenarios.

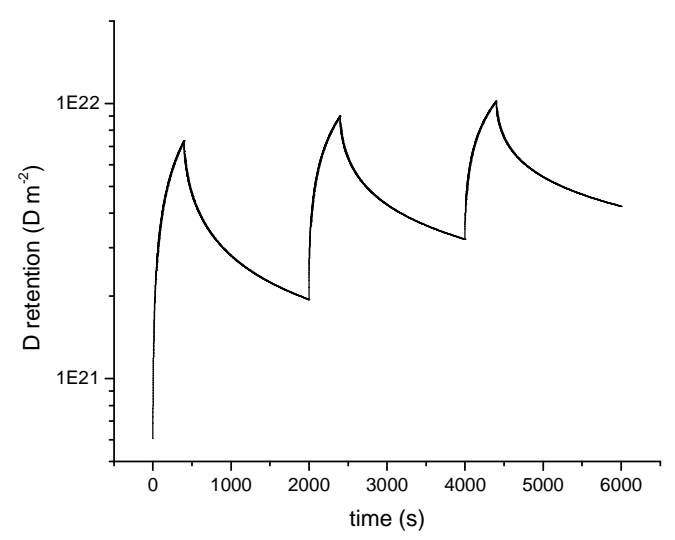

Figure 5. Deuterium retention vs. time

3.1 Tungsten as first wall material with $1 \mathrm{~Hz}$ ELM pulses 
Figures 6 and 7 show D retention as a function of the time during the $400 \mathrm{~s}$ pulse duration with and without ELM effect. From Fig. 6, the retention level is about $9.9 \times 10^{21} \mathrm{Dm}^{-2}$ without thermal spike pulses produced by ELMs. The effect of ELMs with $1 \mathrm{~Hz}$ frequency reduces the retention to about $2.3 \times 10^{21} \mathrm{Dm}^{-2}$. Therefore the ELMs helped to reduce D retention by about $77 \%$ of that of the no-ELM case) due to the thermal spikes induced at each second of the operation. Because the retention ripples cannot be seen clearly at the 400 -s scale, we plotted a 10-s graph in Fig. 7 to show details of the ELM effects.

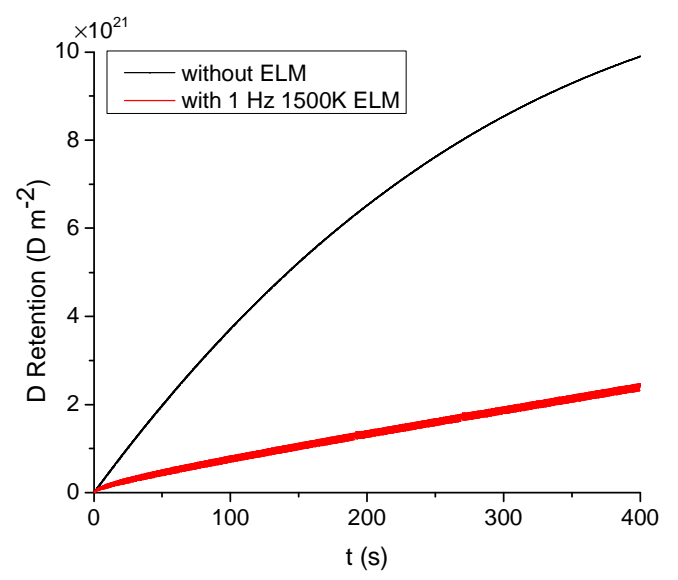

Figure 6. First wall simulation with and without $1 \mathrm{~Hz}$ ELM thermal pulses

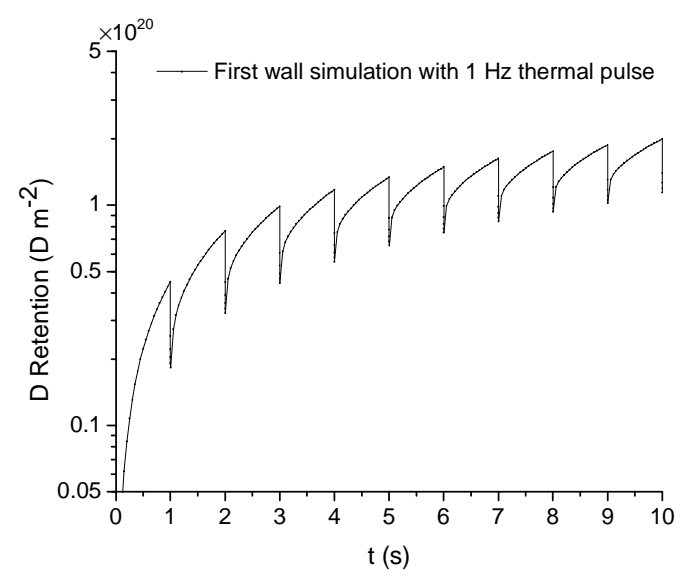

Figure 7. First wall simulation with $1 \mathrm{~Hz}$ thermal pulses (closer look)

\subsection{Tungsten as first wall material with $10 \mathrm{~Hz}$ ELM operation}

Figure 8 and 9 show D retention as a function of time during the $400 \mathrm{~s}$ pulse duration with and without the effect of $10 \mathrm{~Hz}$ ELMs. The $10 \mathrm{~Hz}$ thermal spikes reduced the retention to about $2.1 \times 10^{21} \mathrm{Dm}^{-2}$. In this case, the higher-frequency ELMs reduced D retention by about 79\% from the no-ELMs case. The 10-s result is plotted in Fig. 9 to provide a clear view of the $10 \mathrm{~Hz}$ ELM effects. The retention amount for the $10 \mathrm{~Hz}$ ELM case is not significantly less than that of the $1 \mathrm{~Hz}$ ELM case. One reason is that the $500 \mu$ s ELM time duration is too short for thermal conduction. Another factor is that the $1 \mathrm{~Hz}$ frequency removes most of the mobile deuterium; thus, to remove more retained $\mathrm{D}$, one must wait for the trapped deuterium to become mobile again and move to the surface so it can be recombined and removed. Therefore, there is no significant difference between the $1 \mathrm{~Hz}$ and $10 \mathrm{~Hz}$ cases. We compare the results of $1 \mathrm{~Hz}$ - and $10 \mathrm{~Hz}$-ELMs in Fig. 10. 


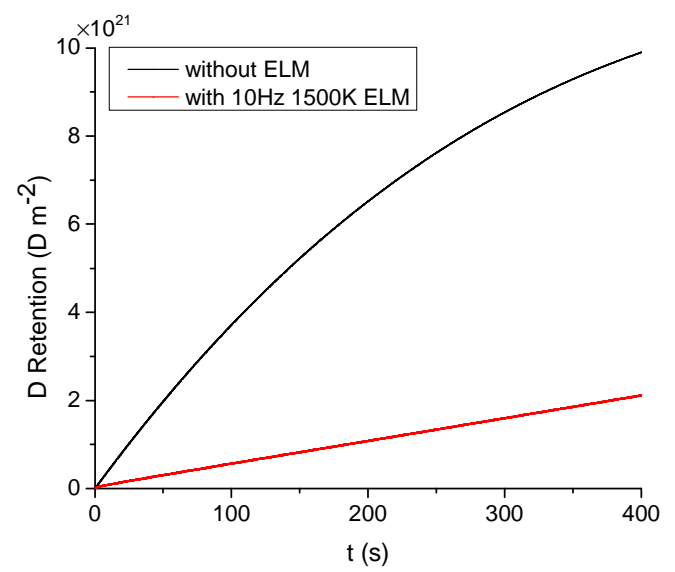

Figure 8. First wall simulation with and without $10 \mathrm{~Hz}$ ELM thermal pulses

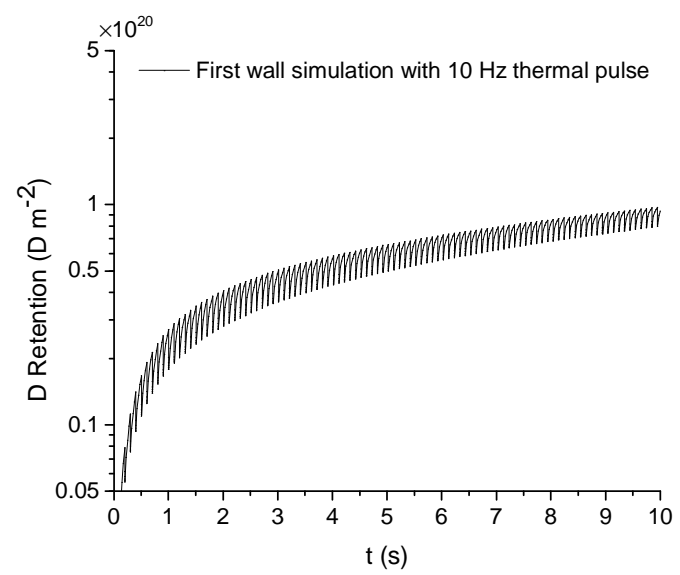

Figure 9. First wall simulation with $10 \mathrm{~Hz}$ ELM thermal pulses (closer look)

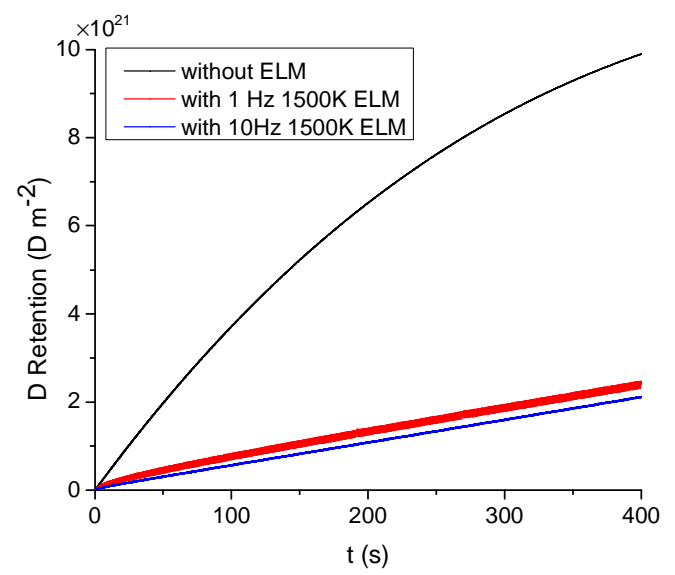

Figure 10. First wall simulation with $1 \mathrm{~Hz} / 10 \mathrm{~Hz}$ and without ELM thermal pulses

\subsection{Tungsten as divertor material with $1 \mathrm{~Hz}$ ELM pulses}

During simulation of the divertor region, ion flux is $10^{24} \mathrm{~m}^{-2} \mathrm{~s}^{-1}$ with deuterium energy at about $15 \mathrm{eV}$ [14]. Temperature at the surface is assumed to be $1000 \mathrm{~K}$ with a $1 \mathrm{~Hz} 500 \mu$ s pulse achieving transient temperature of around $2000 \mathrm{~K}$, while the temperature for the rear side is set at $380 \mathrm{~K}$. Figure 11 shows that D retention is about 
$1.9 \times 10^{25} \mathrm{Dm}^{-2}$ for operation without thermal spike pulses of ELMs. During the $10 \mathrm{~Hz}$ ELMs operation, the D retention is about $2.1 \times 10^{24} \mathrm{Dm}^{-2}$. Therefore the ELM mode of operation in the divertor reduced the retention to about $11 \%$, retention with thermal transient spikes. The retention in the divertor area is lower than at the first wall, which corresponds to our intuition that higher surface and ELM temperatures will result in reduced deuterium retention.

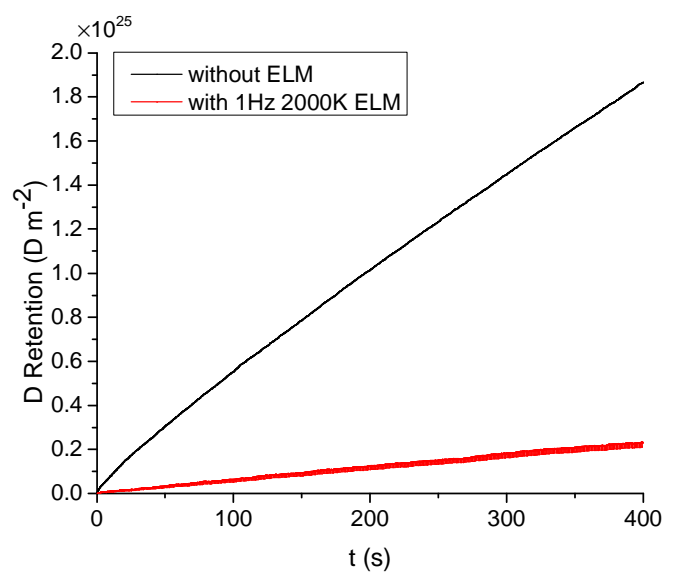

Figure 11. Retention in divertor with and without $1 \mathrm{~Hz}$ ELM operation

\subsection{Overnight cleaning}

We simulated potential overnight cleaning after the previous $400 \mathrm{~s}$ pulse duration with $1 \mathrm{~Hz}$ ELM operation for both the divertor and first wall. The cleaning temperature used, e.g., using laser scanning was $2300 \mathrm{~K}$ for $10 \mathrm{~ms}$. Figure 12 shows that the first wall area retention before cleaning is $9.9 \times 10^{21} \mathrm{Dm}^{-2}$ and that after cleaning is reduced to $1.0 \times 10^{20} \mathrm{Dm}^{-2}$ i.e., to about $1 \%$ of its value. Therefore about $99 \%$ of the deuterium is removed during the active heating using laser pulses of $10 \mathrm{~ms}$ duration for the cleaning process. Figure 13 shows that divertor area retention before the active heating was $1.9 \times 10^{25} \mathrm{Dm}^{-2}$ and after the cleaning is reduced to $1.6 \times 10^{25} \mathrm{Dm}^{-2}$, or about $85 \%$ of its value, which means that only about $15 \%$ of the deuterium is removed by cleaning. The reason for the first wall's higher removal rate is that more deuterium is retained in the tungsten, and also that the percentage of mobile deuterium is higher and therefore easier to remove than is the trapped deuterium.

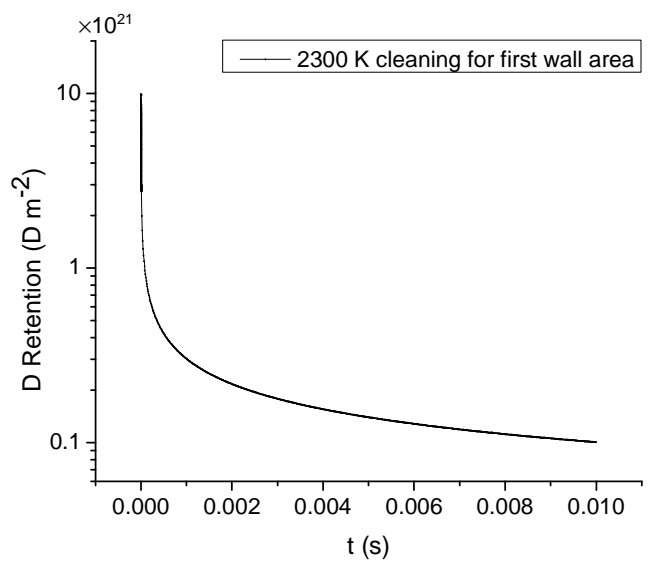

Figure 12. Effect of successive heating on first wall area at $2300 \mathrm{~K}$ for $10 \mathrm{~ms}$ after a $400 \mathrm{~s}$ operational pulse 


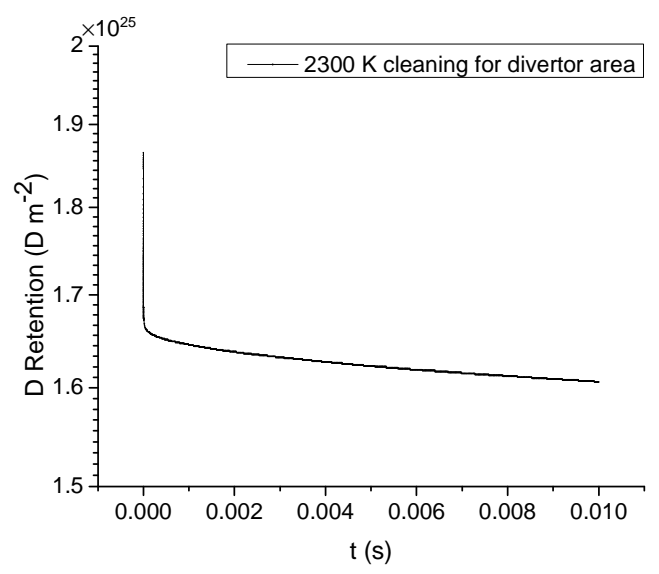

Figure 13. Effect of successive heating on divertor area at $2300 \mathrm{~K}$ for $10 \mathrm{~ms}$ after a $400 \mathrm{~s}$ operational pulse

4. Discussion and conclusions

Minimizing tritium retention in plasma-facing components (PFCs) of future fusion reactors is a key concern. The mobile and trapped inventory in PFCs will depend on many parameters and conditions. The parameters needed for more accurate prediction of fuel retention that we used in our analysis can be classified into three groups. The first group contains parameters from the experimental conditions used for benchmarking, such as temperature, deuterium energy, flux, fluence, tungsten type, and measurement method. The values in this group are basically accurate. The second group contains parameters that have more or less acceptable accuracy and include diffusion coefficients and physical description of major controlling equations. Although theoretically speaking, single-crystal tungsten and polycrystalline tungsten may have different diffusion coefficients because deuterium can diffuse along grain boundaries or dislocations, the effect of this factor may be small compared to that of the temperature influence on diffusivity and is neglected in this research for simplification. Also, activation energy can differ by temperature zone, but we retain and fix its value as the widely used $0.39 \mathrm{eV}$. The major equations in our simulation also assume that all detrapped atoms become mobile and do not consider the probability of a detrapped atom leaving a trap site to become trapped again. However, this effect is expected to be of second order and therefore is small compared to the other two terms in the trapped equation, so the major equations still represent the physical system well. However, more studies and analysis are required for a firm conclusion.

The third group contains parameters that need more analysis and more basic theoretical study, such as the surface recombination coefficient, radiation-enhanced diffusion, trap-density profile, and ion-induced stress value. For example, we used a surface recombination coefficient (critical to hydrogen isotope release) that is given by

$$
K_{r}=3.2 \times 10^{-15} \exp \left(\frac{-1.16}{k T}\right)
$$

which is the same as in the Anderl et al. work [16]. However, Anderl et al. measured surface recombination over the temperature range of $690-825 \mathrm{~K}$; if we extrapolate that to the $300-500 \mathrm{~K}$ region, the value may be too small in comparison to the modeling result from Garcia-Rosales et al. [17] and Franzen et al. [18] Therefore, both the preexponential factor and the activation energy need further research [19]. In addition, the recombination coefficient is very sensitive to surface conditions and impurity contents that can significantly reduce its value and therefore increase wall retention.

Another example is the radiation-enhanced diffusion value. We used the expression from Ogorodnikova et al. given by [7]

$$
D(x, t)=D_{m}\left(1-\left(1-\frac{D}{D_{m}}\right) \exp \left(-\frac{(1-r) I_{0} \psi(x) \eta t}{\mu_{m}}\right)\right)
$$

where $D(x, t)$ is the radiation-enhanced diffusion; $D_{m}$ is the maximum coefficient; $\mathrm{D}$ is the bulk diffusion coefficient; $\mathrm{r}$ is the reflection coefficient; $\psi(x)$ is the depth distribution of ion-induced defects; $\eta$ is the defect creation rate, and $I_{0}$ is the incident flux. The radiation-enhanced diffusion value is about ten times larger than the bulk value when fluence is about $10^{23} \mathrm{D} / \mathrm{m}^{2}$ and twenty times larger when fluence is about $10^{24} \mathrm{D} / \mathrm{m}^{2}$. In this 
research we used ten times the bulk diffusion coefficient value as the surface diffusion value, however further studies coupled with more experiments are needed to obtain a more accurate value.

A relation between the oversaturated deuterium concentrations versus stress profile needs to be developed. However, this is not directly and spatially proportional to each other and there should be some shift in depth, such as deuterium concentration $(\mathrm{x}) \propto$ stress $(\mathrm{x}+\Delta \mathrm{x})$. Currently molecular dynamics (MD) simulation methods are being utilized to developing such relationship.

In conclusion, we have developed models to study hydrogen isotope behavior in metallic plasma-facing components in fusion reactors. From our previous simulation [3], we have benchmarked our models with various experiments and simulation results. Our results are in good agreement with recent experimental data and other work. Therefore, our results have been used to simulate and predict hydrogen isotope inventory and wall recovery during realistic fusion reactor operation.

From the results, we can conclude that edge-localized modes (ELMs) can reduce deuterium inventory; also, higher ELM temperature and higher frequency both reduce retention levels. About $20 \%$ of the deuterium remains in tungsten in the first wall condition and about $10 \%$ in the diverter condition compared to the no-ELM case. For cleaning processes, e.g., using laser-pulsed heating with $2300 \mathrm{~K}$ material temperature and $10 \mathrm{~ms}$ duration, about $15 \%$ of the deuterium is removed from the divertor and $99 \%$ is removed from the first wall.

However, further work is required in four respects. First, one must verify the details of the coefficients values for the specific material/alloy used for the design, as discussed in the previous section. Second, more details should be added to the model in the future to account for surface-moving boundaries from sputtering erosion, surface roughness, and impurity effects. There is also the need to account for surface build-up in redeposition areas and erosion from plasma transient events due to vaporization/splashing during abnormal events, as well as implementation during moving boundaries due to surface erosion process. Other mechanisms involving irradiation and diffusion-enhanced processes need to be considered in this model. Additional mechanisms such as surface segregation, impurity effect on surface recombination, chemical sputtering, mixing of materials, and neutron damage all play important roles in fusion reactor operation; they therefore must be considered in detail in the future development of more extensive and integrated simulation coupled and benchmarked with relevant experiments to reactor conditions.

Acknowledgment

This work is partially supported by the U.S. Department of Energy, Office of Fusion Energy Sciences, and the National Science Foundation (PIRE) project.

\section{References}

[1] Tsitrone, E., Journal of Nuclear Materials, 2007. 363: p. 12-23.

[2] Perevezentsev, A.N., et al., Journal of Nuclear Materials, 2008. 372(2-3): p. 263-276.

[3] $\mathrm{Hu}, \mathrm{A}$. and A. Hassanein, Journal of Nuclear Materials, 2014. 446(1-3): p. 56-62.

[4] Haasz, A.A., et al., Physica Scripta, 2007. T128: p. 55-59.

[5] Skinner, C.H., et al., Fusion Science and Technology, 2008. 54(4): p. 891-945.

[6] Alimov, V.K., J. Roth, and M. Mayer, Journal of Nuclear Materials, 2005. 337(1-3): p. 619-623.

[7] Ogorodnikova, O.V., J. Roth, and M. Mayer, Journal of Applied Physics, 2008. 103(3).

[8] Hindmarsh, A.C., et al., Acm Transactions on Mathematical Software, 2005. 31(3): p. 363-396.

[9] Ogorodnikova, O.V., J. Roth, and M. Mayer, Journal of Nuclear Materials, 2003. 313: p. 469-477.

[10] Ahn, T.M., 1984, Department of Nuclear Energy,Brookhaven National Laboratory.

[11] Ohmi, T., A.T. Yokobori, Jr., and K. Takei, Diffusion in Solids and Liquids Vii, 2012. 326-328: p. 626631.

[12] Hassanein, A. and T. Sizyuk, Nuclear Fusion, 2008. 48(11).

[13] Sizyuk, T., A. Hassanein, and M. Ulrickson, Fusion Engineering and Design, 2013. 88(3): p. 160-164.

[14] Roth, J., et al., Journal of Nuclear Materials, 2009. 390-91: p. 1-9.

[15] Alimov, V.K. and J. Roth, Physica Scripta, 2007. T128: p. 6-13.

[16] Anderl, R.A., et al., 1992. 21(2): p. 745-752.

[17] GarciaRosales, C., et al., Journal of Nuclear Materials, 1996. 233: p. 803-808.

[18] Franzen, P., et al., Journal of Nuclear Materials, 1997. 241: p. 1082-1086.

[19] Causey, R.A., Journal of Nuclear Materials, 2002. 300(2-3): p. 91-117. 
Reviewer \#2: On the Gorsky effect:

With the availability of the actual input parameters the referee was able to reproduce the Gorsky effect calculations.

However these only exist under the extreme and quite unusual conditions the authors have applied:

Authors agree the condition is extreme and unusual since normally hydrogen will diffuse into metal and will not form blister on metal surface. However we can see that the D concentration in the implantation zone greatly exceeds the solubility limit and stresses the matrix lattice until plastic deformation with the formation of blisters on the surface under this low energy high flux/fluence irradiation condition.

* High extremely localized stress field with high gradients, constant in time and independent on the amount of implanted $\mathrm{H}$ which supposedly produces the stress?!?

* Low diffusion coefficients

Authors have replied carefully in the following.

If the authors would have used the more commonly accepted value for the solute diffusion coefficient by Frauenfelder (as is also used in the quoted ref 7 and 9 by Ogorodnikova), which has a prefactor 3 orders of magnitude higher than the value by Franzen used by the authors, the Gorsky effect disappears: The smoothing effect of the gradient driven diffusion wins over the peaking by the stress gradient. The value by Franzen is known to be influenced by trapping and thus is probably not a good representation for the solute diffusion coefficient.

First, the diffusion coefficient of $D=4.1 \times 10^{-7} \exp \left(-0.39 / \mathrm{kT}^{2} \mathrm{~m}^{2} \mathrm{~s}^{-1}\right.$ is used in other simulation work such as Ogorodnikova et al.[1], C. H. Sang et al.[2] and R. H. Ning et al.[3] However this value by Frauenfelder [4] in fig. 1 is measured in temperature range from $1200 \mathrm{~K}$ to $2400 \mathrm{~K}$. But when we focus on benchmarking with Alimov et al.[5] for temperature range around $300 \mathrm{~K}$ to $800 \mathrm{~K}$ this will cause Frauenfelder's result to be too large in this region for our purpose. With this fact the value $D=1.5 \times$ $10^{-10} \exp (-0.39 / \mathrm{kT}) \mathrm{m}^{2} \mathrm{~s}^{-1}$ is selected which is between the modeling values by Franzen et al.[6] $\left(1.5 \times 10^{-10} \exp (-0.25 / \mathrm{kT}) \mathrm{m}^{2} \mathrm{~s}^{-1}\right)$ and Garcia-Rosales et al.[7] ( $3.5 \times 10^{-11} \exp (-0.39 /$ $\mathrm{kT}) \mathrm{m}^{2} \mathrm{~s}^{-1}$ ).

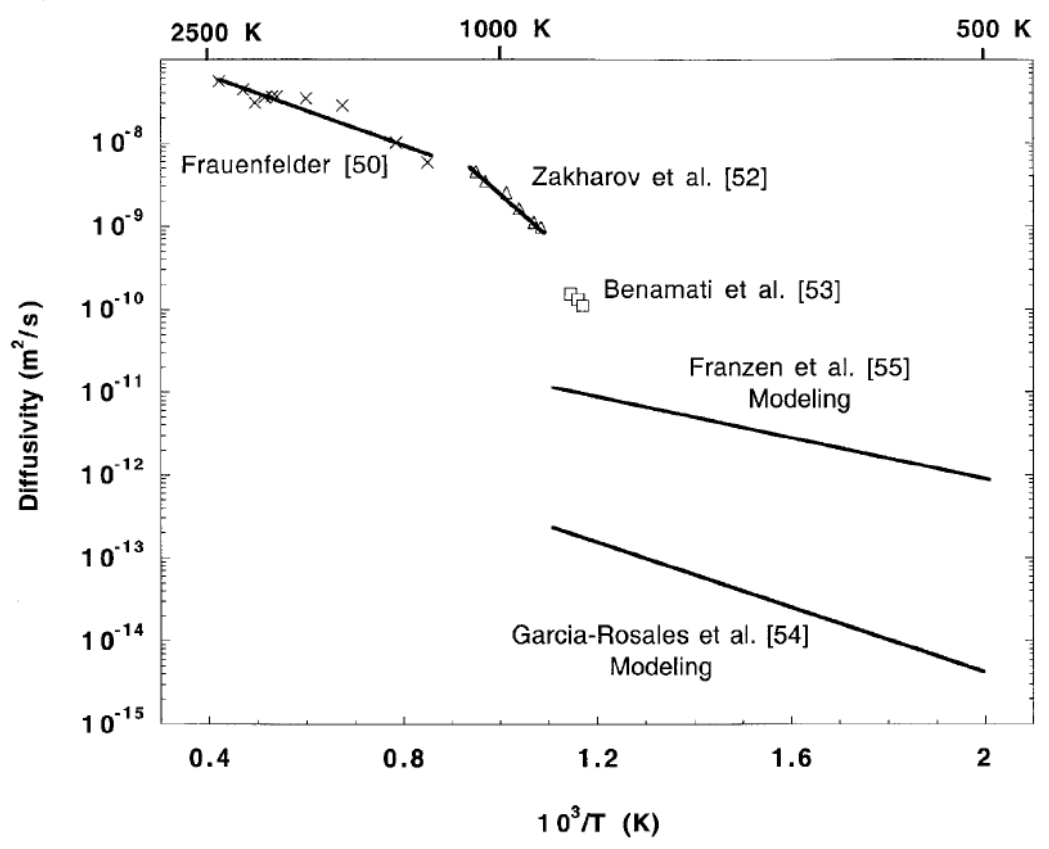


Figure 1: Hydrogen diffusivity in tungsten[8]

Moreover, in the results given by Alimov et al.[9], it can be observed that deuterium gradually diffuse into bulk as temperature goes higher. However, if we perform the simulation using $D=4.1 \times$ $10^{-7} \exp (-0.39 / \mathrm{kT})$ and only the source and diffusion term as in equation (1), the phenomena of gradual diffusion cannot be seen.

$\frac{d C}{d t}=G+D \frac{\partial^{2} C}{\partial x^{2}}$

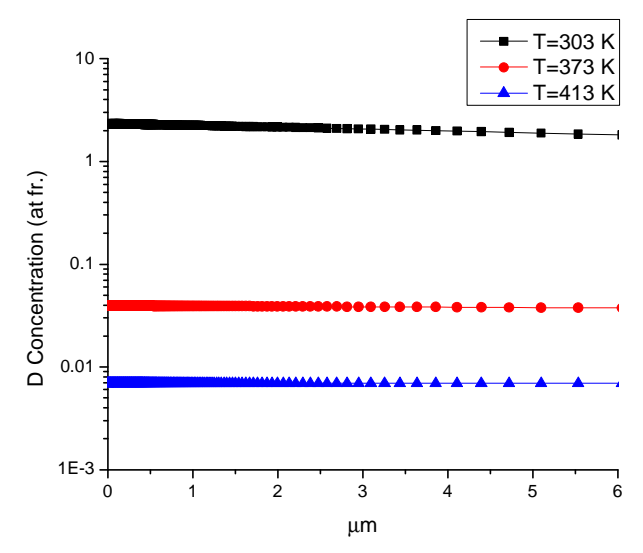

Figure 2: Deuterium distribution at higher diffusion coefficient

Figure 2 shows the diffusion result using $D=4.1 \times 10^{-7} \exp \left(-\frac{0.39}{\mathrm{kT}}\right)$ for equation (1). The diffusion process is very fast that the concentration becomes almost flat near the surface region. If further including trap sites, the deuterium distribution would be very similar to the trap distribution because the mobile deuterium concentration is same everywhere. And this is a possible reason why there is no obvious temperature difference in other simulation work. Since the diffusion coefficient can be composed by segments in different temperature region, the value $D=1.5 \times 10^{-10} \exp (-0.39$ / kT) $\mathrm{m}^{2} \mathrm{~s}^{-1}$ is selected which is between the modeling values by Franzen et al.[6] $\left(1.5 \times 10^{-10} \exp (-0.25 / \mathrm{kT}) \mathrm{m}^{2} \mathrm{~s}^{-1}\right)$ and Garcia-Rosales et al.[7] ( $3.5 \times 10^{-11} \exp (-0.39 /$ $\left.\mathrm{kT}) \mathrm{m}^{2} \mathrm{~s}^{-1}\right)$. Figure 3 shows the diffusion result with $D=1.5 \times 10^{-10} \exp (-0.39 / \mathrm{kT})$ for equation (1). With the lower diffusion coefficient, the gradually diffusion processes can be simulated.

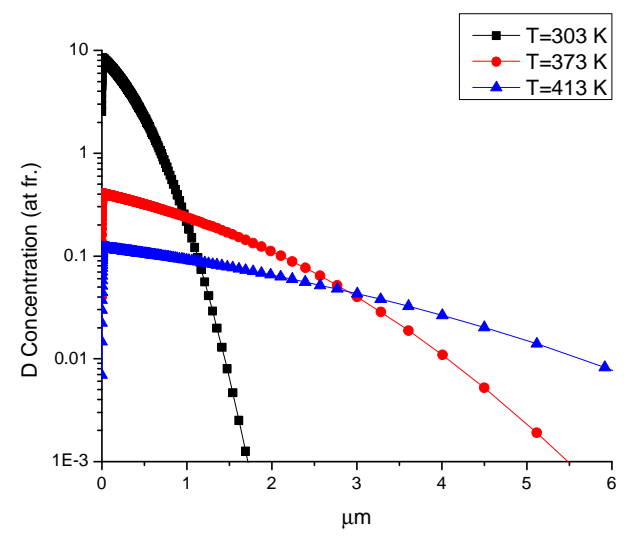

Figure 3: Deuterium distribution at lower diffusion coefficient 
Secondly the assumption of a strongly peaked gauss shaped stress profile is hard to understand. The authors say that a normal distribution is the simplest choice. However one could equally well argue for a linear stress gradient: Compressive stress in the oversaturated implantation region and tensile stress in bulk balancing the forces similar to beam bending. So why such a strange shape, independent on the local amount of implanted D?

First the authors would like to point out there is no tensile strain in this D->W case compared to classical Gorsky effect. The bending scenario of Gorsky effect can result one side of compressive strain and one side of tensile strain, however in our case, we proposed the massive amount of deuterium implantation on the surface will squeeze, distort the neighbor lattice and produce a high compressive stress area beneath the oversaturated implantation peak which the compressive stress will gradually decrease/relax as extending into the bulk. Therefore only compressive strain exists.

Secondly, indeed Gaussian distribution shape is not a delicate way to describe the stress, but linear shape is even worse. As we have explained in the first part, what we are looking for is some profile that can mathematically gradually reduce as depth axis goes to infinite.

Finally, authors agree there should be a relation between oversaturated deuterium concentration versus the stress profile. However, it is not directly proportional to each other at same location as $(x) \propto P(x)$; in fact, there should be some shift in depth, such as $D(x) \propto P(x+\Delta x)$. Authors are currently working on developing this relationship by using molecular dynamics simulation.

Also there appears to be a fundamental error in the derivation of equation 3 from equation 2 in their ref 3 where their code is described. They lack the mixed derivatives and thus a linear stress field gradient in their model would result in no Gorsky effect at all. I.e. it appears they cannot simulate the original beam bending Gorsky effect with their code if the equations given in ref 3 are what they use in their code. This error in the equations of course is of little consequence for the assumed extreme condition of a normal distributed stress field.

So in summary the authors Gorsky effect only exists under rather extreme conditions and whether they are justified is questionable. Also there appears to be a fundamental error in their stress gradient driven diffusion expression.

The conditions treated here are extreme and justifies the use of this effect. In addition, the fact that diffusion process is not only related to concentration but also affected by stress and temperature is not created by authors, it is clearly stated in every derivation in many applications. One for example may check the mass diffusion analysis part in the abaqus user manual as a quick reference.

Last but not least the dip they create by the Gorsky effect can equally well be reproduced by taking into account the loss of $D$ from the surface during cool down of the sample after implantation: Without any new affects or extreme assumptions.

Authors did include the loss of $D$ from the surface by taking surface recombination coefficient into account.

Therefore the referee would suggest that the authors skip the Gorsky effect in their paper and re-do their calculations with more commonly accepted parameters. (e.g. Frauenfelder diffusion)

For results without our assumptions and justifications, the reviewer can find such results in other published researches that didn't explain the experimental data well. The authors think lattice strain and local stress field play an important role in deuterium distribution is the reason why we do this research. 
Of course additional work is needed for future analysis and planned experimental work in our CMUXE facilities.

On the surface temperature evolution:

The step like temperature evolution is a simple representation of the effect of an ELM. But why is it a step function? The authors claim they solve the heat transport equation and this step function does not look like a solution to the heat transport equation. Also why is the inter ELM temperature so low? For ITER the target temperature should at least be around 1200K and more like 2000K at the outer target. More information about their temperature modeling is therefore required. (e.g. the Power influx time evolution $\mathrm{W} / \mathrm{m} 2$ )

The reviewer requested last time "a plot of at least the surface temperature vs time for a single ELM like event.", therefore authors plot the surface temperature verse time as fig. 4 . The temperature rise during ELMs and disruptions is determined by the various parameters of the escaping plasma particles during these instability events. The Co-author is leading these activities worldwide for the last 20 years.

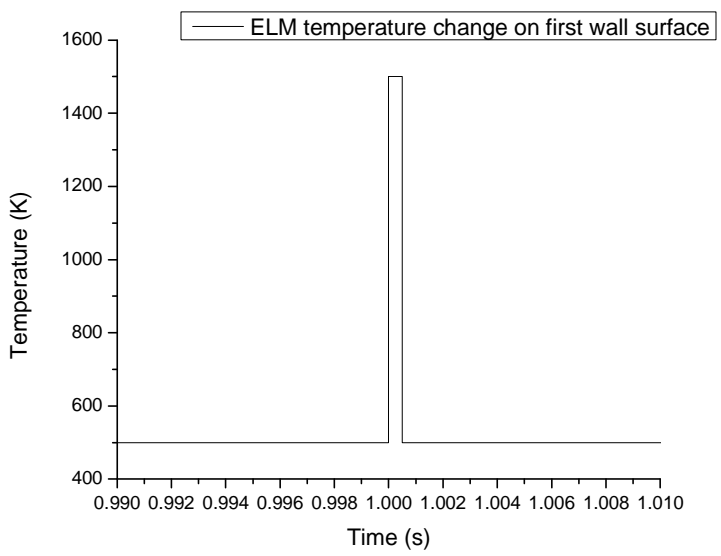

Figure 4.

In order to simplify and simulate the deuterium inventory amount and recovery by cleaning processes, we have to add the heat equation into the system of differential equations. The equation is shown below, where $T$ is the temperature distribution and $\kappa$ is the thermal diffusivity.

$\frac{d T}{d t}=\kappa \frac{\partial^{2} T}{\partial x^{2}}$

There are several different designs for the ITER inner surface tiles, which are list in table 1 . Since we focus on deuterium behavior in tungsten, the condition we model is the monoblock tungsten tile design. Here CFC stands for carbon fibre-reinforced carbon composite and the alloy under CFC is $\mathrm{CuCrZr}$ heat sink. 
Table 1: different tile configuration[10]

\begin{tabular}{|c|c|c|}
\hline & CFC surface & Tungsten surface \\
\hline 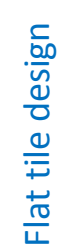 & & \\
\hline $\begin{array}{l}\frac{1}{00} \\
\frac{0}{0} \\
\frac{0}{0} \\
\text { ㅇ } \\
\frac{0}{0} \\
\frac{0}{0} \\
\frac{0}{2} \\
\text { ¿ }\end{array}$ & & \\
\hline
\end{tabular}

The values used in simulation for flux, deuterium energy, etc. were taken from refernece[11]. The flux for first wall is assumed to be $10^{20} \mathrm{~m}^{-2} \mathrm{~s}^{-1}$ and flux for divertor $10^{24} \mathrm{~m}^{-2} \mathrm{~s}^{-1}$; deuterium energy for first wall can be between 8-300 eV. However we choose it to be $200 \mathrm{eV}$ since we did numerous benchmarking for $200 \mathrm{eV}$ with Alimov's work[9], and the deuterium energy for divertor is $15 \mathrm{eV}$. The temperature for the first wall surface is assumed to be $500 \mathrm{~K}$ and divertor surface temperature is $1000 \mathrm{~K}$, also the ELM thermal pulse for first wall is assumed to be $1500 \mathrm{~K}$ and ELM temperature for divertor is $2000 \mathrm{~K}$. In order to decide what scale and boundary condition to use, we look into reference[12, 13] as in figure 5 and 6 to help decide on the initial and boundary conditions. Although the materials are not exactly the same as we are simulating, we can still implement the module dimesion and coolant temperature as our inputs. The initial condition for deuterium concentration is zero everywhere and temperature distrubute uniformly with $380 \mathrm{~K}$ at beginning. The total length for simulating deuterium concentration is $200 \mu \mathrm{m}$ and for temperature is $15 \mathrm{~mm}$. The boundary condition for concentration equations is $\mathrm{J}=\mathrm{K}_{\mathrm{r}} \mathrm{C}(0)^{2}$ at front side and $\mathrm{D}=0$ or $\mathrm{J}=0$ at rear side; for heat equation the front side temperature may vary according to ELM and temperature are rear side is constant at 380K.

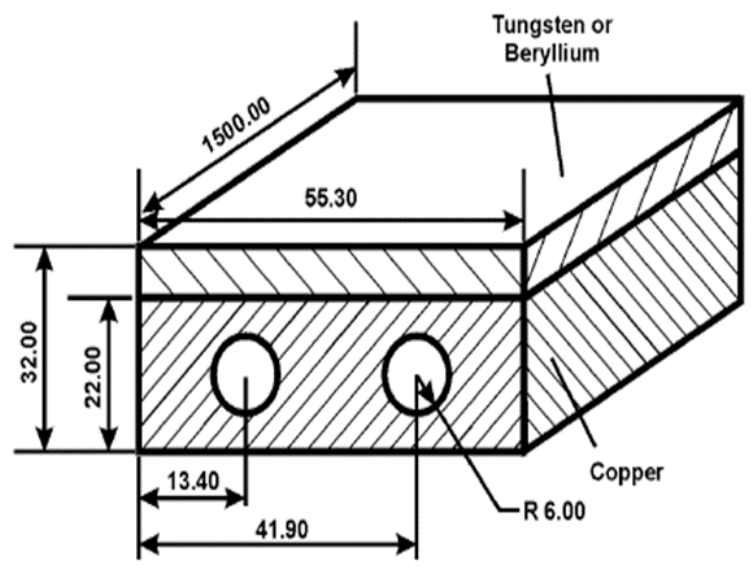


Figure 5: Schematic illustration of a ITER-like divertor module design[12]

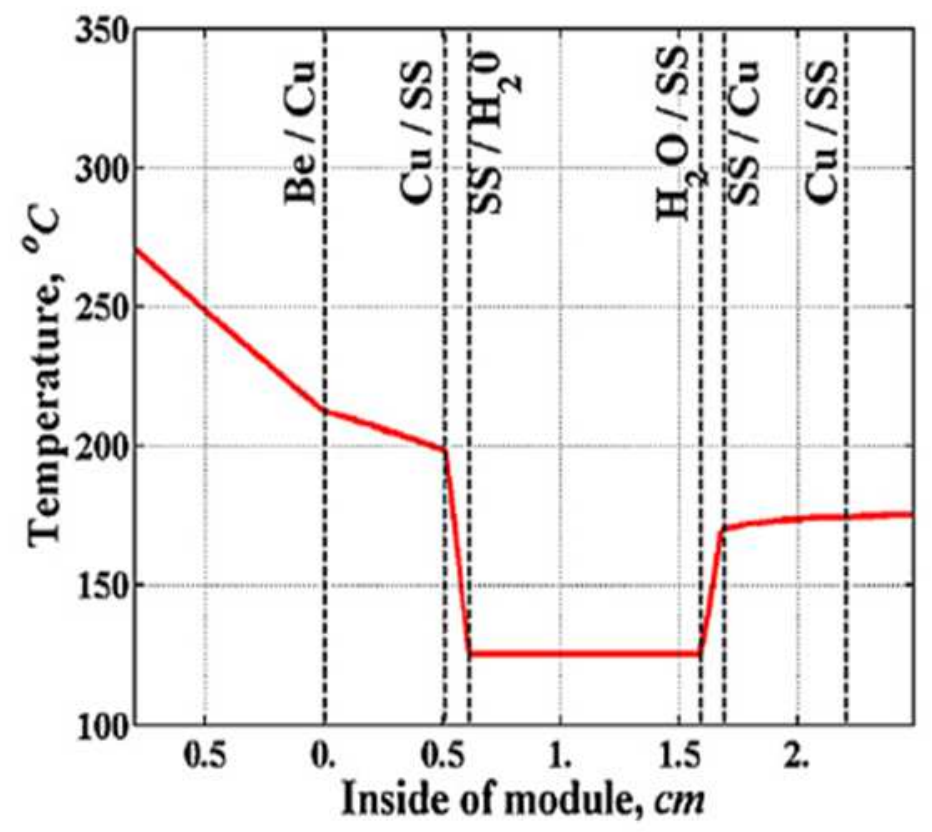

Figure 6: steady-state temperature distribution inside the module with $8 \mathrm{~mm} \mathrm{Be}$ armor and with SS tube, $1 \mathrm{MW} / \mathrm{m} 2$ steady-state heat flux (inlet water temperature of $\left.115^{\circ} \mathrm{C}\right)[13]$

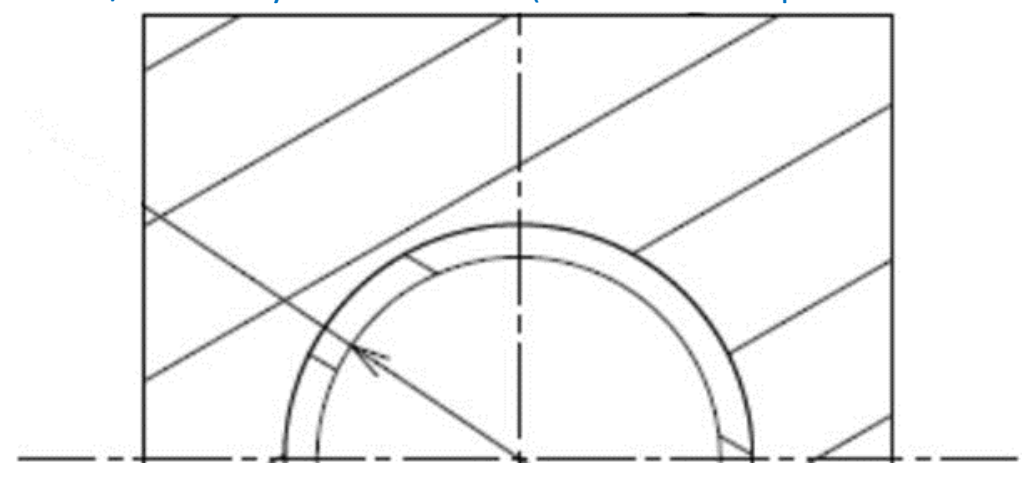

Figure 7: half crossection of tile module

In conclusion, the temperature at the bottom of figure 7 consists to be $380 \mathrm{~K}$ and the temperature at the top of figure 4 varies as listed in table 2 .

Table 2: parameter values

\begin{tabular}{|l|l|l|}
\hline & First wall & Divertor \\
\hline Flux & $10^{20} \mathrm{~m}^{-2} \mathrm{~s}^{-1}$ & $10^{24} \mathrm{~m}^{-2} \mathrm{~s}^{-1}$ \\
\hline D energy & $200 \mathrm{eV}(8-300 \mathrm{eV})$ & $15 \mathrm{eV}$ \\
\hline Surface T & $500 \mathrm{~K}$ & $1000 \mathrm{~K}$ \\
\hline ELM pulse T & $1500 \mathrm{~K}$ & $2000 \mathrm{~K}$ \\
\hline
\end{tabular}


On the trap profile evolution:

The authors make a point about the trap profile evolution which they base on a trap profile evolution model originally developed for ion beam implantation. Apart from the fact that this is probably not applicable to the particle influx from an ELMy H-mode, what is really lacking is the effect of annealing of the defects during the high temperatures. Therefore this needs to be discussed in paper or the calculation repeated with constant trap profiles, stating explicitly that the trap profile evolution is ignored. This also ties into the question about the inter ELM temperature which in reality will be probably higher than what is assumed in their calculations.

So in summary the paper is on the right track now and could be accepted after further major modifications.

The deuterium depth profile we benchmarked with Alimov et al[5], which after polishing the samples were annealed at $1573 \mathrm{~K}$ for 3.5 hours in a vacuum chamber with a background pressure of $\sim 2 * 10^{-4} \mathrm{~Pa}$ during annealing.

The trap profile description and parameters we implemented are from Ogorodnikova et al[1] work, all their samples were annealed at $1573 \mathrm{~K}$ for 3 hours in vacuum with a background pressure of $2 * 10^{-4} \mathrm{~Pa}$ before implantation.

Since both annealing conditions are similar, it is straight forward to relate the experimental and simulation parameters together.

The trap profile will evolute with ion beam implantation, but will not influenced by annealing when we simulate for ELM. This point of view is added in the paper. Other trap details are stated as following.

The increase in trap density can be written as:

$$
\frac{\mathrm{dC}_{\text {Trap }}}{\mathrm{dt}}=(1-\mathrm{r}) \cdot \text { flux } \cdot \psi(x) \cdot \eta \cdot\left(1-\frac{\mathrm{C}_{\text {Trap }}}{C_{\text {Trap }}^{0}}\right)
$$

where $\mathrm{C}_{\text {Trap }}$ is the trap density; $\mathrm{r}$ is the reflection coefficient; $\psi(x)$ is the depth distribution of ioninduced defects; $\eta$ is the defect creation rate and $C_{\text {Trap }}^{0}$ is the maximum defect concentration. After integration with time $t$, we obtain the trap density as following:

$\mathrm{C}_{\text {Trap }}(\mathrm{x}, \mathrm{t})=C_{\text {Trap }}^{0}\left(1-\exp \left(-(1-r) \cdot\right.\right.$ flux $\left.\left.\cdot \psi(x) \cdot \eta \cdot t / C_{\text {Trap }}^{0}\right)\right)$

(4)

Table 3

\begin{tabular}{|l|l|l|l|}
\hline Detrap E & Trap types & Maximum trap density & Defect production rate \\
\hline \multirow{2}{*}{$0.85 \mathrm{eV}$} & Low energy intrinsic traps & $C_{\text {Trap }}^{0}=3 \times 10^{-5}$ at fr. & \\
\cline { 2 - 4 } & Ion-induced traps & $C_{\text {Trap }}^{0}=0.005$ at fr. & $\eta=2 \times 10^{-3}$ \\
\hline \multirow{2}{*}{$1.45 \mathrm{eV}$} & high energy intrinsic traps & $C_{\text {Trap }}^{0}=1 \times 10^{-5}$ at fr. & \\
\cline { 2 - 4 } & lon-induced traps & $C_{\text {Trap }}^{0}=0.01$ at fr. & $\eta=1 \times 10^{-3}$ \\
\hline
\end{tabular}

[1] O. V. Ogorodnikova, J. Roth, and M. Mayer, "Ion-driven deuterium retention in tungsten," Journal of Applied Physics, vol. 103, Feb 12008. 
[2] C. Sang, X. Bonnin, M. Warrier, A. Rai, R. Schneider, J. Sun, et al., "Modelling of hydrogen isotope inventory in mixed materials including porous deposited layers in fusion devices," Nuclear Fusion, vol. 52, Apr 2012.

[3] R. H. Ning, Y. G. Li, W. H. Zhou, Z. Zeng, and X. Ju, "AN IMPROVED CLUSTER DYNAMICS MODEL FOR HYDROGEN RETENTION IN TUNGSTEN," International Journal of Modern Physics C, vol. 23, Jun 2012.

[4] Frauenfe.R, "SOLUTION AND DIFFUSION OF HYDROGEN IN TUNGSTEN," Journal of Vacuum Science \& Technology, vol. 6, pp. 388-\&, 19691969.

[5] V. K. Alimov, J. Roth, and M. Mayer, "Depth distribution of deuterium in single- and polycrystalline tungsten up to depths of several micrometers," Journal of Nuclear Materials, vol. 337, pp. 619-623, Mar 12005.

[6] B. Cantelli, F. M. Mazzolai, and M. Nuovo, "INTERnAL FRICTION DUE TO LONG-RANGE DIFFUSION OF HYDROGEN IN NIOBIUM (GORSKY EFFECT)," Physica Status Solidi, vol. 34, pp. 597\&, 19691969.

[7] C. GarciaRosales, P. Franzen, H. Plank, J. Roth, and E. Gauthier, "Re-emission and thermal desorption of deuterium from plasma sprayed tungsten coatings for application in ASDEXupgrade," Journal of Nuclear Materials, vol. 233, pp. 803-808, Oct 1996.

[8] R. A. Causey, "Hydrogen isotope retention and recycling in fusion reactor plasma-facing components," Journal of Nuclear Materials, vol. 300, pp. 91-117, Feb 12002.

[9] V. K. Alimov and J. Roth, "Hydrogen isotope retention in plasma-facing materials: review of recent experimental results," Physica Scripta, vol. T128, pp. 6-13, Mar 2007.

[10] " "313th PFMC Workshop / 1st FEMaS Conference, Tutorial Course, Rosenheim," ed, 2011.

[11] J. Roth, E. Tsitrone, T. Loarer, V. Philipps, S. Brezinsek, A. Loarte, et al., "Tritium inventory in ITER plasma-facing materials and tritium removal procedures," Plasma Physics and Controlled Fusion, vol. 50, Oct 2008.

[12] A. Hassanein and T. Sizyuk, "Comprehensive simulation of vertical plasma instability events and their serious damage to ITER plasma facing components," Nuclear Fusion, vol. 48, Nov 2008.

[13] T. Sizyuk, A. Hassanein, and M. Ulrickson, "Thermal analysis of new ITER FW and divertor design during VDE energy deposition," Fusion Engineering and Design, vol. 88, pp. 160-164, Mar 2013.

In summary, we thank the referees for their comments and stimulating discussions. Hope these modifications/clarifications helped answer the questions. 